\title{
allergica 2002 - nehmen Allergiekongresse überhand?
}

B ochum - vom 13. bis zum 16. März 2002 tagte die Deutsche Gesellschaft für Allergologie und klinische Immunologie auf dem Campus der RuhrUniversität. Kaum zwei Monate später, vom 24. bis zum 26. Mai 2002 organisierte die Messe Frankfurt am Main mit Unterstützung namhafter Kollegen eine Tagung, die einerseits auf die betroffenen Laien zielte, mit einer Ausstellung zu entsprechenden Produkten für Allergiker, andererseits aber auch Symposien über fachspezifische Themen einbezog, die in Form von Fortbildungsvorträgen derzeitige Auffassungen zu zahlreichen Themen den mehr oder minder interessierten Fachkollegen vermittelten.

Angaben über die definitive Teilnehmerzahl liegen den Unterzeichnern noch nicht vor - der Blick durch die Messehallen "Neue Wege der Wis- sprach aber sensvermittlung sind für sich, ebengefragt - nicht zuletzt so der Blick in auch, um die Öffent- die Hörsäle. lichkeit stärker über die Dennoch: es aktuellen Probleme des war ja nur der Gesundheitswesens erste Versuch. zu informieren." Daher muss sicherlich das Konzept noch einer kritischen Überprüfung unterzogen werden, bevor eine endgültige Bewertung der Sinnhaftigkeit einer allergica möglich sein wird.

Interessant auch für die Allergologen ist zweifellos die Öffnung für die interessierte Öffentlichkeit, für Patienten und deren Angehörige. Als für diese Zielgruppe neuartiges Informationsforum wurde die allergica 2002 zumindest zeitweise offensichtlich auch angenommen, wie die Kommentare der Aussteller zeigten Patientenorganisationen, Hersteller von Produkten für Allergiker, Pharmafirmen, Gerätehersteller, Allergiekliniken.

Interessant auch die Referate für Laien, zu Themen wie Medikamentenkunde, Allergie und Beruf, Allergie und Umwelt, Hyposensibilisierung, Asthmaschulung, Rehabilitation, Asthma und Sport, Teufelszeug Kortison, Hausstaubmilben etc. Diese Themen stießen

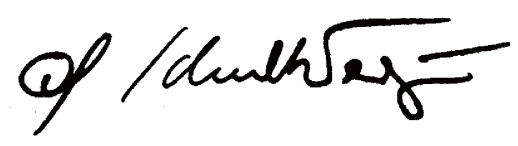

Prof. Dr. G. Schultze-Werninghaus auf großes Interesse und haben sicherlich Zukunft.

Anders die Fortbildungsvorträge für Ärzte, hier war das Interesse sehr wechselhaft. Ob also der Zwitter aus Wissenschaft und Öffentlichkeitsarbeit in dieser Form Bestand haben kann, sollte ernsthaft überprüft werden. Wichtig kann die allergica vor allem auch für den Transport des Wissens und der aktuellen Probleme in die Medien werden - dabei könnte die Verbindung mit professionellen Veranstaltern, wie der Messe Frankfurt, sicherlich hilfreich sein.

Neue Wege der Wissensvermittlung sind gefragt - nicht zuletzt auch, um die Öffentlichkeit stärker über die aktuellen Probleme des Gesundheitswesens zu informieren. Wahrscheinlich wäre auf diese Weise mehr zu bewegen Stichworte Disease-Management-Programme - als durch Bemühungen aus Fachkreisen allein.

Da das Konzept noch sozusagen im Erprobungszustand ist, sollten wir die Chance zur Mitgestaltung nutzen. Auch Ihre Meinung ist gefragt!

Mit den besten Grüßen,

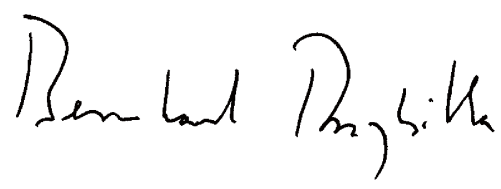

Prof. Dr. B. Przybilla 